\title{
Observations and a Model of NGC 2610
}

\author{
J. Patrick Harrington \\ Department of Astronomy, University of Maryland, College Park, MD 20742, USA \\ email: jph@astro.umd.edu
}

\begin{abstract}
While photoionization codes have been carefully intercompared, a fundamental need for clean tests against real nebulae remains. NGC 2610 is a high-excitation planetary nebula which, even at HST resolution, is smooth and symmetric. Helium is $\mathrm{He}^{++}$throughout this nebula, which has a high electron temperature $(20,000 \mathrm{~K})$ resulting in strong UV lines. It is the best object we know of to test the performance of photoionization codes without the complication of low ionization knots or filaments. Its large angular diameter ( $40^{\prime \prime}$ ) allows spatial gradients to be observed. In 2001 and 2003, we obtained HST STIS long-slit observations to test against models. Observed lines cover wavelengths from $1240 \AA$ to $6563 \AA$. Interstellar reddening is small. The [O III] $\lambda 4363$ line flux is needed to determine the electron temperature. This line proved unmeasurable in the HST spectra, so we obtained ground-based (Kitt Peak 4-m) observations at the same slit position as the HST data.

We have constructed photoionization models of this nebula, and compare one with our observations. Most lines are in good agreement. The most discordant line is [Ne IV] $\lambda 2424$, which is observed to be twice as strong as predicted. Collisional excitation of $\mathrm{H}^{0}$ is the most important coolant, responsible for $30 \%$ of the total. Observations of the Balmer decrement in this nebula can put useful constraints on $\mathrm{H}^{0}$ collision strengths.
\end{abstract}

\section{Introduction}

It is generally assumed that photoionization codes are well tested, and indeed different codes are in substantial agreement for benchmark models. We argue that there is still a need for clean comparisons of photoionization codes with real nebulae, especially highexcitation objects, where atomic data may be uncertain and the transfer of line radiation is an important issue. NGC 2610 is the best object we know of to test the performance of photoionization codes without the complication of low ionization knots or filaments. With this in mind, we obtained HST observations of this nebula.

\section{Observations}

Long-slit spectra of NGC 2610 were obtained with the HST STIS in 2001 and 2003. The observed lines cover wavelengths from $1240 \AA(\mathrm{N}$ V) to $6563 \AA(\mathrm{H} \alpha)$. Because this nebula has a low surface brightness, the slit was opened to 2 arcsec. This degrades the wavelength resolution, in some cases to $\sim 50 \AA$, but the lines are still separated and measurable.

For the stronger UV lines, the fluxes are in reasonable agreement with the only other UV spectra - IUE whole-nebulae fluxes obtained by Feibelman (2000) in 1981. It appears that IUE overestimated the strengths of some weaker lines.

In principle, the best measure of interstellar extinction should be obtained from the ratio of $\mathrm{He}$ II $\lambda 1640$ to He II $\lambda 4686$, due to the long wavelength baseline. For our HST spectra, we found that this ratio slightly exceeds the theoretical value. Earlier studies found $c=0.05 \pm 0.02$ for the extinction (Shaw \& Kaler (1989)). We conclude that the extinction is negligible and use the theoretical $\lambda 1640 / \lambda 4686$ to adjust the absolute scale of UV vs. visual fluxes. Our HST fluxes, relative to $\mathrm{H} \beta=100$, are given in the Table 1 . 
Table 1. Model (CLOUDY06) vs. Observations

\begin{tabular}{lcccllccc}
\hline Ion & $\lambda(\AA)$ & Model & HST & $\|$ & Ion & $\lambda(\AA)$ & Model & HST \\
\hline$N V$ & 1240 & 51. & 44. & $\|$ & {$[N e V]$} & 3426 & 80. & 80. \\
$O I V]$ & 1402 & 112. & 128. & $\|$ & {$[N e I I I]$} & 3869 & 30. & 27. \\
$N I V]$ & 1486 & 64. & 77. & $\|$ & $H \gamma$ & 4340 & 47. & 47. \\
$C I V$ & 1549 & 1770. & 1850. & $\|$ & {$[O I I I]$} & 4363 & 9.9 & 11.3 \\
$H e I I$ & 1640 & 778. & 763. & $\|$ & $H e I I$ & 4686 & 105. & 103. \\
$O I I I]$ & 1665 & 33. & $50 ? ?$ & $\|$ & $H \beta$ & 4861 & 100. & 100. \\
$C I I I]$ & 1909 & 338. & 553. & $\|$ & {$[O I I I]$} & 5007 & 317. & 327. \\
{$[N e I V]$} & 2424 & 141. & 305. & $\|$ & $H \alpha$ & 6563 & 292. & $260 ?$ \\
\hline
\end{tabular}

The [O III $\lambda 4363$ line flux is needed to determine the electron temperature. This line proved unmeasurable in the HST spectra, so we obtained ground-based (Kitt Peak 4-m) observations at the same slit position as the HST data. This is the source of the $\lambda 4363$ flux in Table 1 . The the [O III $] \lambda 5007 / \lambda 4363$ temperature varies with projected slit position from $21,000 \mathrm{~K}$ to $18,000 \mathrm{~K}$ as we go from near the center to the edge of the nebula, as expected from the photoionization models.

\section{A Photoionization Model}

As a first cut at a photoionization model of NGC 2610, we present a simple, uniform density, thick spherical shell calculated using CLOUDY06. The stellar flux is a Rauch halo model, $\mathrm{T}=142,000 \mathrm{~K}, \log \mathrm{g}=6.5,2600 L_{\odot}$. The density is $n_{H}=180 \mathrm{~cm}^{-3}$, from 0.12 - 0.227 pc. Gas abundances are (H:He:C:N:O:Ne $\left.=10^{6}: 11000: 120: 17: 150: 25\right)$. In Table 1 we compare the observed line fluxes integrated along the slit to the total fluxes from the model. Future models will address the variations along the slit. The chemical abundances are well below solar and this contributes to the high electron temperature of this object.

A very interesting aspect of this nebula is the role played by the collisional excitation of hydrogen. $\mathrm{H}^{0}$ is the leading coolant, providing $~ 30 \%$ of the energy loss; $\mathrm{H} \alpha$ and $\mathrm{H} \beta$ have major collisional contributions. But note that $\mathrm{H}^{0} \mathrm{n}=1 \rightarrow \mathrm{n}=3,4,5$ collision strengths are uncertain: see the study of G135.9+55.9 by Péquignot \& Tsamis (2005).

\section{Conclusions}

While the agreement between the model and observations seems generally satisfactory, there are some indications of problems. The most obvious of these is the [Ne IV] $\lambda 2424$, which is over twice the strength predicted by the model, even though the [Ne III] and $[\mathrm{Ne} \mathrm{V}]$ lines are in agreement. We point out that a careful study of the Balmer series in this nebula should place useful constraints on $\mathrm{H}^{0}$ collision strengths.

\section{Acknowledgements}

Support for Program No. HST-GO-09178.01-A was provided by NASA through a grant from the Space Telescope Science Institute, which is operated by AURA, Inc. under NASA contract NAS5-26555.

\section{References}

Feibelman, W.A. 2000, PASP 112, 861

Péquignot, D. \& Tsamis, Y.G. 2005, A\&A 430, 212

Shaw, D. \& Kaler, Y.G. 1989, ApJS 69, 495 